\title{
Local distortions and volume changes in semiconductors: donors in silicon
}

\author{
A M Stoneham $\dagger$, B Pajot $\ddagger$ and H R Schober£ \\ + Theoretical Physics Division, Harwell Laboratory, Oxon OX11 0RA, UK \\ ¥ Groupe de Physique des Solides de l'ENS, Tour 23, Université Paris 7, 75251 Paris \\ Cedex 05, France. \\ $\S$ Institut für Festkörperforschung, Kernforschungsanlage Jülich, Postfach 1913, D-5170 \\ Julich, Federal Republic of Germany.
}

Received 16 May 1988

\begin{abstract}
Experiments giving impurity-induced lattice distortion can measure quite distinct quantities. In particular EXAFS (extended x-ray fine structure) measures nearest-neighbour distances, whereas both volume changes and recent spectroscopic data measure long-range displacements. The relationship between the two depends strongly on the inter-atomic potential. We analyse this for impurities in silicon by adopting a variety of current potentials.

There is a significant apparent contradiction between the EXAFS results and the other experiments for all of the inter-atomic potentials. This problem may be associated with the high oxygen concentrations of Czochralski crystals used in the EXAFS study.
\end{abstract}

\section{Displacements observed near defects in semiconductors}

The substitution of an impurity for a host atom in a crystal causes other atoms to be displaced from their sites in the perfect host. These displacements can be observed in several ways: from the macroscopic volume change or change in $x$-ray lattice parameters (see, e.g., Siegert et al (1984), Cohen and Taylor (1987) p 1132), from experiments that monitor the displacements over many lattice sites (as in the high-resolution spectroscopy of Pajot and Stoneham (1987)) or from the experiments like EXAFS (extended x-ray absorption fine structure) which can measure the distance between an impurity and its nearest neighbours (e.g. Erbil et al 1987).

The relationship between the displacements derived from these methods is straightforward only when elasticity theory can be used, i.e. when long-wavelength displacements alone are involved. The main results are given for isotropic elasticity by Stoneham ((1985) \$ 8.3.3). There are complications, but no significant problems, for anisotropic elasticity (Stoneham (1985) p 776, Bass et al (1984)). However, elasticity theory does not suffice to relate EXAFS data to volume changes. This is partly because of the shorter wavelength displacements involved, and partly because the elasticity analysis usually assumes that the defect forces that drive the displacements are applied at what is effectively a point, i.e. that the displacements are observed at much larger distances than those at which the forces are applied. This is clearly incorrect for nearest-neighbour displacements. The discrete atomic structure and the proper inter-atomic forces must 
be used in modelling the relationship between longer- and shorter-range deformations.

In the present paper we examine the data for donors in silicon, and compare these with predicted relations between volume changes $\Delta V$ for a large but finite crystal and the nearest-neighbour displacement $u$ for a range of inter-atomic potentials. We shall also remark on the consequences of defects influencing the local force constants though, for the main part, we shall assume the sole effect of the impurity is to apply radial outward forces $F$ to its nearest neighbours.

\section{Elasticity theory and beyond}

A useful reference point is the result from second-order isotropic elasticity. We may use equations (8.3.64) and (8.3.70) of Stoneham (1985) to give the radial displacement $u$ of the nearest neighbours (which are at distance $a$ in the perfect crystal) in terms of $\Delta V$ :

$$
u / a=\left(\Delta V / 4 \pi a^{3}\right) \frac{1}{3}\left(C_{11}+2 C_{12}\right) / C_{11}
$$

or in terms of the defect forces $F$ :

$$
u / a=F /\left(3 \pi a^{2} C_{11}\right) \text {. }
$$

With data for Si $\left(C_{11}=1.657 \times 10^{12} \mathrm{dyn}^{-2}, C_{12}=0.639 \times 10^{12} \mathrm{dyn}^{-2}\right.$ and $a=$ $2.35 \AA$ ) we find for isotropic elasticity theory (equation (1))

$$
u(\AA)=0.044 F(\mathrm{eV} \AA)^{-1}
$$

and, for anisotropic elasticity theory (Deutz and Schober (1983), using $C_{44}=0.80 \times 10^{12}$ dyn $\mathrm{cm}^{-2}$ ):

$$
u(\AA)=0.058 E\left(\mathrm{eV}^{-1}\right)
$$

where the defect force is given in units appropriate to recent atomistic calculations (see, e.g., Scheffler et al 1985).

Turning to discrete lattice models, we may use dynamic methods (see, e.g., Larkins and Stoneham 1971) or static methods (see, e.g., Schober 1977) to find the nearestneighbour displacement per unit force $F$; this is, of course, the $\mathrm{A}_{1}$ symmetry response function of Larkins and Stoneham. We have done these calculations both for Keating potentials and for several other valence force potentials listed in the compilation by Torres and Stoneham (1985).

The Keating potential is defined by two parameters, $\alpha$ and $\beta$. The $\alpha$ parameter cancels out of the relationship between $u$ and $\Delta V$ and, in any case, varies rather little among the several Keating potentials listed. In all our calculations we have used Keating's original value of $\alpha=3.03 \mathrm{eV} \AA^{-2}$ and varied $x=\beta / \alpha$. The value of this ratio ranges quite widely from author to author. We find

$$
u(\AA)=\left(2.30 / \theta^{2}\right) F\left(\mathrm{eV} \AA^{-1}\right)
$$

with $\theta$ the effective frequency in $10^{13} \mathrm{rad} \mathrm{s}^{-1}$, which can be fitted (Stoneham et al 1988) to the analytic form:

$$
\theta^{2}=\left(61.020 x^{2}+106.907 x+22.967\right) /(x+0.440) .
$$

The two extreme values of $x$ are for the potentials of Baraff $e t$ al (1980) (x=0.0911) and of Keating (1966) $x=0.2838$. If we write

$$
\Delta V=C 4 \pi a^{2} u
$$


and $a=2.35 \AA$, then values of $C$ are 1.694 for isotropic elasticity, 2.013 for the potential of Baraff et al (1980) and 2.588 for the Keating (1966) potential. Clearly it is a poor approximation to take $C=1$ (i.e. to assume $\Delta V$ to be the volume of the shell between the spheres through the relaxed and unrelaxed nearest neighbours). These results also show that a nearest-neighbour displacement estimated from a known $\Delta V$ is rather sensitive to which inter-atomic potential is used to relate them: elasticity theory is not enough. This problem is additional to the complications of the actual displacement pattern, for which the displacements deviate from the simple $r^{-2}$ behaviour of elasticity theory (see Bass et al (1984) or Scheffler (1987)) both in angular dependence and shortrange dependence or $r$.

We may extend these results to the various other valence force potentials. Convenient expressions can be obtained using equation (4) or the analogous form

$$
\Delta V\left(\AA^{3}\right)=D \theta^{2} u(\AA) .
$$

and $D=2.23$ for frequencies $\theta$ in $10^{13} \mathrm{rad} \mathrm{s}^{-1}$. The frequencies $\theta$ are listed by Stoneham et al (1988) for a range of valence force potentials. Equation (7) alone, however, shows that softer potentials ( $\theta$ small) will lead to larger local displacements for a given $\Delta V$. Expression (7) allows us to look too at the effects of local changes in force constants. Values of $\theta$ are available for vacancies in silicon, modelled by either removal of only radial force constants to the defect site (Lannoo and Allan 1982) or by removing both bond-bending and bond-stretching forces. These removals of forces near vacancies lead to reductions in $\theta$ for vacancies that can be dramatic (see Larkins and Stoneham 1971), so $u$ may be enhanced by up to an order of magnitude for a given volume change. Such enhancements are not serious possibilities for simple substitutional donors, as can be verified from infrared spectra. Thus the recent careful analysis of Si:P by Leigh and Sangster (1984) indicates a change in local force constant (and hence an upper bound on the enhancement of $u$ ) of a mere $20 \%$.

\section{Links between different experiments}

We may now turn to the values for donors in Si. Pajot and Stoneham used a spectroscopic method, based on the species-dependent deviation of the excited states of donors from their effective-mass values. These results (table 1) proved to be well in line with those from Pauling radii and are also roughly in line with high-concentration data (Siegert $e t$ al 1984), though there would surely be clustering in the high-concentration systems. The case $\mathrm{Si}$ : As is the only one that has also been studied by EXAFS (Erbil et al 1986).

Table 1. Estimates of $\Delta V / V_{0}$ for donors in silicon

\begin{tabular}{lllll}
\hline & P & As & Sb & \\
\hline Pajot and Stoneham (1987) & -0.08 & +0.04 & +0.17 & (Anisotropic elasticity) \\
Siegert et al (1984) & -0.2 & -0.01 & +0.042 & \\
Pauling radii & -0.17 & +0.03 & +0.57 & \\
EXAFS & & 0.35 & & (Isotropic elasticity analysis) \\
& & +0.54 & & (Keating potential analysis) \\
\hline
\end{tabular}


The EXAFS data yield a small change $0.06 \pm 0.02 \AA$ in nearest-neighbour distances and a still smaller change in second-neighbour displacement $(0.01 \pm 0.02 \AA)$. The values of $4 \pi a^{2} u / V_{0}$ (with $V_{0}=(8 / 3 \sqrt{ } 3) a^{3} \simeq 20 \AA^{3}$ being the volume per atom in the perfect silicon lattice) is 0.21 , giving values of $\Delta V / V_{0}$ ranging from 0.35 (isotropic elasticity theory) to 0.54 (Keating potential). However we analyse the EXAFS data, the deduced volume change is about an order of magnitude larger than values based on the longerrange displacement field measurements, whether direct measurements of $\Delta V$ or from spectroscopic data.

It is not easy to see how the EXAFS data can be reconciled with other data. Whilst the local force constants could be softened, the necessary reduction would be such as to give an unrealistically low frequency corresponding to $\theta \approx 3.5$. Could there be a problem with the assumption of a fully harmonic lattice? This assumption has led to problems in the past, and even to predictions of the wrong sign of the formation volume in some ionic crystals. The way this can be rectified has been discussed by Flynn (1971), Schober and Ingle (1980), Lidiard (1981), Gillan (1983) and Stoneham (1983). The expressions relevant here are given by Stoneham (1983) and involve the Grüneisen constant and the photo-elastic constants of silicon. The corrections are quadratic in the defect forces, and prove negligible for the defects considered here; they do not resolve the difference between the EXAFS data and the volume change.

There is another possible basis for the differences between the volume changes for $\mathrm{Si}$ : As derived from the different types of experiment, and this involves the role of oxygen impurity. The spectroscopic determination of the relative volume changes associated with P, As and Sb donor atoms in silicon reported by Pajot and Stoneham (1987) were obtained with samples grown by the float zone (FZ) method, where the oxygen contamination remains generally below $5 \times 10^{15}$ atoms $\mathrm{cm}^{-3}$. The concentrations of oxygen in the S-, Se- and Te-doped samples used by the same workers were $\simeq 1 \times 10^{16}$, $\simeq 2 \times 10^{17}$ and $3 \times 10^{17}$ atoms $\mathrm{cm}^{-3}$, respectively. However, in contrast to the low levels available from the float zone method, silicon crystals grown from a melt contained in a silica crucible (Czochralski or CZ crystals) have an interstitial oxygen content in the $10^{18}$ atoms $\mathrm{cm}^{-3}$ range, that is two orders of magnitude bigger. The average distance between oxygen atoms $\simeq 100 \AA$, still much larger than the distance between two silicon atoms bridged by an oxygen atom (this distance is $3.19 \AA$ as compared with $2.35 \AA$ for a normal $\mathrm{Si}-\mathrm{Si}$ bond length) but comparable with effective $\mathrm{Bohr}$ radii. How far does the distortion extend, and does it modify the relative volume change associated with a donor atom? $\mathrm{X}$-ray lattice constant measurements, which probe long range effects, failed to reveal any difference in the lattice spacings of $\mathrm{FZ}$ and $\mathrm{CZ}$ silicon crystals (Okada and Tokumaru 1984). However, differences were consistently observed between the positions of the $P$ lines in FZ and $\mathrm{CZ}$ samples in experiments performed recently using Fourier transform spectroscopy at low temperature in the Groupe de Physique des Solides de l'ENS, Paris, i.e. the spectroscopic method does indicate differences apparently due to oxygen.

In the P-doped CZ samples, there is an upward shift of $\simeq 0.3 \mathrm{~cm}^{-1}(\simeq 0.04 \mathrm{meV})$ of the lines arising from transitions from the $1 \mathrm{~s}\left(\mathrm{~A}_{1}\right)$ ground state to those excited states with binding energies $\leqslant 3.12 \mathrm{meV}\left(3 \mathrm{p}_{ \pm}\right.$level $)$. This shift could be accounted for in several ways, perhaps by an increase of the ground state energy by $0.04 \mathrm{meV}$, and could be due to a small change of the elastic constants of the crystal due to the presence of interstitial oxygen. This change should also be the origin of a smaller increase of the binding energies of the $2 \mathrm{p}_{0}, 2 \mathrm{p}_{ \pm}$and ${ }^{3} \mathrm{P}_{0}$ levels detected as a smaller upward shift of the corresponding lines $\left(0.13,0.17\right.$ and $0.23 \mathrm{~cm}^{-1}$, respectively). A less likely explanation of the shifts might be a decrease of the binding energies of the highly excited levels for which the bound 
electron probes the vicinity of the oxygen atoms. These latter effects should become more important for the weakly bound excited states with wavefunctions of large spatial extent.

These shifts give rise to an increase of both the $3 \mathrm{p}_{ \pm}-2 \mathrm{p}_{ \pm}$and $2 \mathrm{p}_{ \pm}-2 \mathrm{p}_{0}\left(\mathrm{~S}_{2 \mathrm{p}}\right)$ splittings for $\mathrm{P}$ in the $\mathrm{CZ}$ material, yielding a measured value for the reduced splitting $\mathrm{S}_{2 \mathrm{p}} /\left(3 \mathrm{p}_{ \pm}-2 \mathrm{p}_{ \pm}\right)$of $1.542 \pm 0.010(1.544 \pm 0.004$ in FZ silicon $)$. This could be interpreted, by comparison with the calculated splitting (Pajot and Stoneham 1987), as a firstneighbour inward distortion which is slightly larger for $\mathrm{P}$ in $\mathrm{CZ}$ silicon than in FZ silicon. However, this depends too on the choice of the $2 \mathrm{p}_{ \pm}$level as one of the two reference levels. The variation of the $3 \mathrm{p}_{ \pm}-2 \mathrm{p}_{ \pm}$splittings with the chemical nature of the donor and with the internal strains of the sample argues for a better choice of a donorindependent reference level.

The $5 \mathrm{p}_{ \pm}$donor level in silicon with an average binding energy of $1.46 \mathrm{meV}$ is a good candidate: the measured differences between the $5 \mathrm{p}_{ \pm}$and $4 \mathrm{p}_{ \pm}$lines for nine isolated donor complexes, either $\mathrm{H}$-like or $\mathrm{He}^{0}$-like, are between 5.86 and $5.93 \mathrm{~cm}^{-1}$. The smallest is for $\mathrm{Sb}$, where the difference goes down to $5.80 \mathrm{~cm}^{-1}$; the value rises to 5.99 $\mathrm{cm}^{-1}$ for the deepest thermal donor TDA ${ }^{0}$. The $5 \mathrm{p}_{ \pm}-2 \mathrm{p}_{0}$ spacings for $\mathrm{P}(\mathrm{FZ}), \mathrm{P}(\mathrm{CZ})$ and As(FZ) are $80.72,80.92$ and $80.97 \mathrm{~cm}^{-1}$, respectively; they show that the splitting observed for $\mathrm{P}$ when in a CZ matrix is closer to that for As in a FZ matrix than it is to the value for $P$ in a FZ matrix. From this, it seems that the $C Z$ matrix leads to an additional outward distortion of the nearest neighbours of a substitutional donor. Now two of the samples used in the EXAFS measurements for As in silicon were obtained on As-implanted $\mathrm{CZ}$ wafers (Cargill 1988). It is possible therefore that part of the lattice distortion discrepancy between the spectroscopic data and the EXAFS results in this case arises from a high oxygen concentration in the wafers used for the As implantation. This possibility could be checked by repeating the EXAFS experiment with low-oxygen FZ substrates. The sensitivity to the presence of oxygen at the $100 \mathrm{ppm}$ level means that especial care should be taken in comparing any a priori theory with experiment.

\section{Acknowledgment}

Some of this work was done as part of the programme of Underlying Research of the UKAEA.

(C) 1988 UKAEA.

\section{References}

Baraff G, Kane E O and Schlüter M 1980 Phys. Rev. B 215662

Bass R, Oates W A, Schober H R and Stoneham A M 1984 J. Phys. F: Met. Phys. 142869

Cargill G S 1988 private communication

Cohen E R and Taylor E N 1987 Rev. Mod. Phys. 591121

Deutz J W and Schober H R 1983 Comp. Phys. Comm. 3087

Erbil A, Weber W, Cargill G S and Boehme R F 1986 Phys. Rev. B 341392

Flynn C P 1971 Z. Naturf, a 2699

Gillan M J 1983 Phil. Mag. A 48903

Keating P N 1966 Phys. Rev. 145637

Lannoo M and Allan G 1982 Phys. Rev. B 254089 
Larkins F P and Stoneham A M 1971 J. Phys. C: Solid State Phys. 4 143, 154

Leigh R S and Sangster M J L 1984 J. Phys. C: Solid State Phys. 17 L305

Lidiard A B 1981 Phil. Mag. 43291

Okada Y and Tokumaru Y 1984 J. Appl. Phys. 56314

Pajot B and Stoneham A M 1987 J. Phys. C: Solid State Phys. 205241

Scheffler M 1987 Physica B + C 146176

Scheffler M, Vigneron J P and Bachelet G 1985 Phys, Rev. B 316541

Schober H R 1977 J. Phys. F: Met. Phys. 71127

Schober H R and Ingle K W 1980 J. Phys. F: Met. Phys. 10575

Siegert H, Becker P and Seyfried P 1984 Z. Phys. B 56273

Stoneham A M 1983 J. Phys. C: Solid State Phys. 16 L925

1985 Theory of Defects in Solids (London: OUP)

Stoneham A M, Torres V J B, Masri P M and Schober H R 1988 Phil. Mag. A 5893

Torres V J B and Stoneham A M 1985 Handbook of Interatomic Potentials III: Semiconductors, Harwell Report AERE R-11942 